\title{
Damage-free Analysis of Biological Materials by Vibrational Spectroscopy in the EM
}

Ondrej Krivanek ${ }^{1}$, Alice Dohnalkova ${ }^{2}$, Zdravko Kochovski ${ }^{3}$, Benedikt Haas ${ }^{4}$, Johannes Müller ${ }^{4}$, Niklas Dellby ${ }^{1}$, Michael Hotz ${ }^{1}$, Andreas Mittelberger ${ }^{1}$, Benjamin Plotkin-Swing ${ }^{1}$, Tracy Lovejoy ${ }^{1}$ and Christoph Koch $^{4}$

${ }^{1}$ Nion Co., Kirkland, Washington, United States, ${ }^{2}$ Pacific Northwest National Laboratory, Richland, Washington, United States, ${ }^{3}$ Institute of Electrochemical Energy Storage, Berlin, Berlin, Germany, ${ }^{4}$ Humboldt-Universität zu Berlin, Berlin, Berlin, Germany

Atomic-resolution compositional analysis in an electron microscope (EM) using electron energy loss spectroscopy (EELS) or energy-dispersive X-ray spectroscopy (EDXS) requires doses of 106 to 1010 e- / $\AA 2$. Doses of this order completely destroy biological samples. Compositional information on biological samples can nevertheless be obtained from frozen hydrated biological samples with worse spatial resolution at much lower doses [1], or at high doses on freeze-dried samples [2, 3]. However, the accompanying radiation damage is severely limiting, and EELS and EDXS microanalysis have not been adopted widely in biological electron microscopy. Vibrational spectroscopy in the electron microscope, introduced 6 years ago [4], can overcome radiation damage by acquiring spectra with the beam positioned a few tens of nm away from a beam-sensitive sample, in the so-called aloof scattering geometry. Spectra due to dipole scattering that bear a close correspondence to FTIR data are then obtained, and the sample experiences essentially no radiation damage [4-9]. At $5 \mathrm{meV}$ energy resolution, which is now readily attainable, aloof vibrational spectra recorded in this way are able to detect even subtle effects such as isotopic substitution of 13C for 12C [6]. Fig. 1 shows background-subtracted vibrational spectra acquired from the sugar trehalose, used by bacteria as an energy source, and from bovine serum albumin (BSA), an animal protein. Features near $400 \mathrm{meV}$ are due to vibrations of hydrogen atoms that typically lie on the outside of the molecules, and these can change as a function of the environment the molecule is in. Features near $200 \mathrm{meV}$ and below are typically due to the vibrations of heavier atoms such as oxygen, carbon and nitrogen, often on the inside of the molecule. They are typically independent of the molecule's environment, and can be used for detecting the presence of different biological molecules in complex samples. Spectra acquired in aloof geometry from outside the sample are not useful for general biological samples, in which the interesting structures occur away from the sample's edge. However, similar spectra can be obtained when the electron beam is placed on a beam-sensitive sample and proceeds to drill a hole [7-9], at doses exceeding around $103 \mathrm{e}-/ \AA$. This arises because the drilled hole is typically $<10 \mathrm{~nm}$ in diameter, and material a few tens of nm away remains largely intact and provides useful vibrational signal. Fig. 2 shows spectra from ice condensed onto a BN flake using a liquid N2 holder. The spectrum obtained from the marked area comes mostly from material 10-30 $\mathrm{nm}$ away from the hole. Had this sample region suffered heavy radiation damage, there would be little hydrogen left. The strong presence of the O-H stretch peak in the spectrum demonstrates that this was not the case. Larger sample areas can be mapped using so called "leap-frog scanning", in which the probe is positioned at well separated locations and made to jump rapidly between them [7-9]. Provided that the spacing between the probe positions is $>10 \mathrm{~nm}$, the material between the holes appears to suffer little radiation damage [9]. In summary, vibrational spectroscopy in the electron microscope promises to probe biological materials in a way that is similar to infrared spectroscopy, at a spatial resolution of about $30-50 \mathrm{~nm}$, while circumventing radiation damage. We are now focused on developing this technique fully, using a side-entry cryotransfer holder in a Nion 
HERMES electron microscope equipped with a fast and efficient EELS direct detector [10], and capable of $5 \mathrm{meV}$ (and better) energy resolution. The results of these investigations will be reported at the meeting.

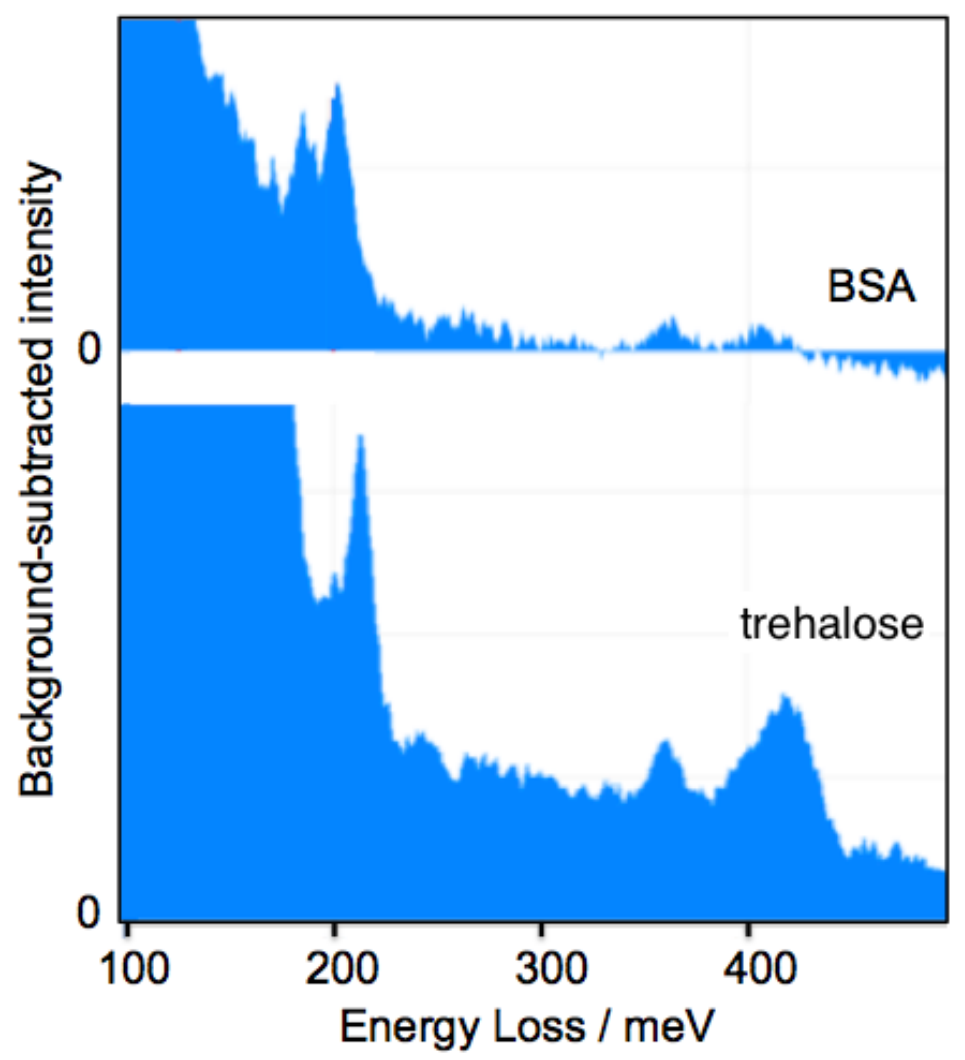

Figure 1. Background-subtracted vibrational EEL spectra from bovine serum albumin (BSA) and the sugar trehalose. 10,000 aloof spectra of $20 \mathrm{~ms}$ each were acquired, aligned in energy, and summed. $\mathrm{E}_{\mathrm{O}}=$ $60 \mathrm{keV}$.
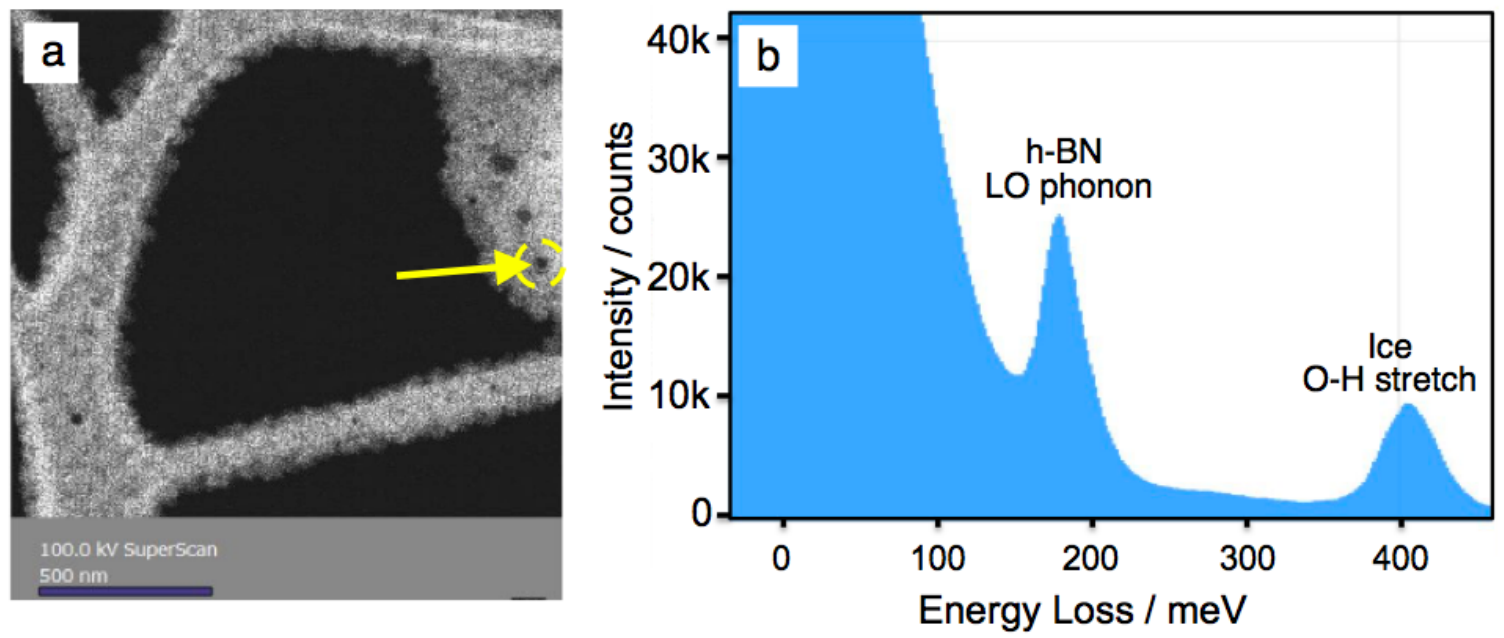

Figure 2. a) Thin layer of ice condensed onto a BN particle on a lacey carbon grid. b) Vibrational spectrum from the circled area in (a). $E_{o}=100 \mathrm{kV}$, single-shot acquisition of $10 \mathrm{~s}$. 


\section{References}

[1] R.D. Leapman and S. Sun, Ultramicroscopy 59 (1995) 71.

[2] J. Feng, A.V. Somlyo and A.P. Somplyo, J. of Micr. 215 (2004) 92.

[3] A.V. Somlyo, H. Shuman and A.P. Somlyo, Methods Enzymol. 172 (1989) 203.

[4] O.L. Krivanek et al., Nature 514 (2014) 209; T. Miata et al., Microscopy 63 (2014) 377.

[5] P. Rez et al., Nature Communications 7 (2016) DOI: 10.1038/ncomms 10945.

[6] J.A. Hachtel et al., Science 363 (2019) 525.

[7] R. Egerton, T. Aoki and P. Crozier, Microsc. Microanal. 22 (Suppl 3, 2016) 960.

[8] R. Egerton, Ultramicroscopy 180 (2017) 115.

[9] P. Rez, private communication (2017).

[10] B. Plotkin-Swing et al., these proceedings (2020). 